\title{
Using factor analysis in beef cattle breeding
}

\author{
Alexey Zelenkov ${ }^{1,2, *}$, Alexey Ermakov², Galina Zelenkova², Ivan Gorlov³, Alexander \\ Pakhomov $^{4}$, Sergei Tresnitskii ${ }^{2}$, Tatyana Derezina ${ }^{2}$ \\ ${ }^{1}$ Ministry of Agriculture and Food of the Rostov region, Krasnoarmeyskaya St., 33, Rostov-on-Don, \\ 344010, Russia \\ ${ }^{2}$ Don State Technical University, sq. Gagarina, 1, Rostov-on-Don, 344010, Russia \\ ${ }^{3}$ The Volga region research institute of manufacture and processing of meat-and-milk products, \\ Rokossovsky St., 6, Volgograd, 400131, Russia \\ ${ }^{4}$ Don State Agrarian University, o. Persianovsky, 346493, Russia
}

\begin{abstract}
Studies of the structure of the interaction of signs with the help of factor analysis is based on the idea of the complex nature of the phenomenon being studied, expressed in the interdependence of individual signs, determined by "internal" hidden causes, forming the specifics of a property, quality. We used the factor analysis method to study the producers' assessments of the quality of the offspring and to test their bullsons for their own productivity of the Kalmyk and Hereford cattle breeds. The analysis included material from the work of pedigree reproducers and plants in the Kalmyk and Hereford breeds of the Rostov Region. The total number of animals included in the treatment was 96 manufacturing bulls and 272 sons bulls. As a result of the studies, the proposed breeding indices on one basis are quite suitable for the preliminary selection of bullcalves in commodity farms or in pedigree farmers for breeding beef cattle. In order to get a final assessment, we compiled new breeding indices, including two (live weight at 8 and 15 months, live weight at 15 months and average daily gain from 8 to 15 months), as well as three characteristics (live weight in 8,15 months and average daily gain). These signs are objective, easy to account. According to the estimates obtained for individual bull-sons, it is possible by summing up the values of new factors to identify the bulls' assessment of the quality of the offspring. Analysis of assessment materials is characterized by high reliability (P> 0.999).
\end{abstract}

\section{Introduction}

Studies of the structure of the interaction of signs with the help of factor analysis is based on the idea of the complex nature of the phenomenon being studied, expressed in the interdependence of individual signs, determined by "internal" hidden causes, forming the specifics of a property, quality. Factor analysis is used to concentrate the source information, including a large number of signs of the analyzed compression phenomenon

\footnotetext{
* Corresponding author: zelenkovalex@rambler.ru
} 
using common factors, expressing in less number of more capacious internal characteristics, i.e. allows you to reduce a large amount of data to possibly whiter than simple concentration with minimal loss of information. The most capacious characteristics obtained, called factors, are not directly measurable. They are behind the scenes of the phenomenon under study, serve as its background and can only be determined as a result of analysis.

The task of factor analysis is to find a simple structure that would accurately reflect and reproduce real, existing in nature dependencies. Moreover, the form and amount of experimental data strongly influence the implementation of the principle of simple structure. A one-sided approach to the selection of signs inevitably leads to a mismatch of the phenomenon being studied. The result of the analysis is determined by the formulation of the entire study.

The goal was to construct a new factor that summarizes the initial information of all the characters from all the signs of testing the bulls for their own productivity, on which the bulls' assessment of the quality of the offspring is based. At the same time, we proceeded from the fact that these signs to some extent correlate with each other. This means that either they mutually define each other, or the relationship between them is determined by some specific quantity that cannot be directly measured. This proposal is in many cases real. The factor analysis model just determines this. Using factor analysis, we determined a value that, like a sponge, absorbed the values of all the signs of an assessment. For analysis, we took the first factor (the main component), which covers the largest set of features, their largest scatter (variance). At the same time, the coverage level of the variance of the signs of the assessment was set at least 95\% [1-12].

\section{Materials and methods}

For research by factor analysis, we took materials on the evaluation of producers on the quality of offspring and tests of their bull sons on their own productivity of the Kalmyk and Hereford breeds. The analysis included material from the work of pedigree reproducers and plants in the Kalmyk and Hereford breeds of the Rostov Region. The study used data from the appraisal of cattle herds of Kalmyk and Hereford breeds. Consolidated valuation statements were submitted to the tribal department of the Ministry of Agriculture and Food of the Rostov Region.

\section{Results and discussion}

The total number of animals included in the treatment was 96 bulls and 272 sons (Table 1).

Table 1. Development and variability of signs for evaluating calves of different breeds by their own productivity, grown on farms of various categories

\begin{tabular}{|c|c|c|c|c|c|c|c|}
\hline \multirow[b]{2}{*}{$\begin{array}{l}\text { Biometric } \\
\text { Constants }\end{array}$} & \multicolumn{2}{|c|}{ Live weight, $\mathrm{kg}$} & \multirow[b]{2}{*}{$\begin{array}{l}\text { The average } \\
\text { daily } \\
\text { increase } \\
\text { from } 8 \text { to } 15 \\
\text { months, } g\end{array}$} & \multirow{2}{*}{$\begin{array}{c}\text { Feed } \\
\text { costs } \\
\text { per } 1 \\
\text { kg of } \\
\text { growth, } \\
\text { feed. } \\
\text { units }\end{array}$} & \multirow[b]{2}{*}{$\begin{array}{c}\text { Intravital } \\
\text { assessment } \\
\text { of meat } \\
\text { qualities, } \\
\text { score }\end{array}$} & \multirow[b]{2}{*}{$\begin{array}{l}\text { Overall } \\
\text { rating, } \\
\text { point }\end{array}$} & \multirow[b]{2}{*}{$\begin{array}{l}\text { Integrated } \\
\text { Index }\end{array}$} \\
\hline & 8 months & $\begin{array}{c}15 \\
\text { months }\end{array}$ & & & & & \\
\hline \multicolumn{8}{|c|}{ KALMYK BREED } \\
\hline \multicolumn{8}{|c|}{$\begin{array}{c}\text { In general by Kalmyk breed } \\
\text { Bulls }(\mathrm{n}=22)-\text { improvers of sons }(\mathrm{n}=51)\end{array}$} \\
\hline 1. $\bar{x} \pm \mathrm{S}_{\mathrm{x}}$ & $188,6 \pm 1,2$ & $387,2 \pm 1,9$ & $938 \pm 8$ & $7,3 \pm .1$ & $50,9 \pm .3$ & $43 \pm .3$ & $102,8 \pm .4$ \\
\hline
\end{tabular}




\begin{tabular}{|c|c|c|c|c|c|c|c|}
\hline 2. $\mathrm{C}_{\mathrm{V}}$ & 9,12 & 6,9 & 11,72 & 13,96 & 7,01 & 10,67 & 4,89 \\
\hline \multicolumn{8}{|c|}{ Bulls $(n=18)-$ neutrals by sons $(n=50)$} \\
\hline 1. $\bar{x} \pm \mathrm{S}_{\overline{\mathrm{x}}}$ & $183,7 \pm 1,5$ & $369,4 \pm 2$ & $849 \pm 7$ & $7,6 \pm .1$ & $50,1 \pm .3$ & $40 \pm .3$ & $100 \pm .3$ \\
\hline 2. $\mathrm{C}_{\mathrm{V}}$ & 10,81 & 7,17 & 11,17 & 14,69 & 8,02 & 10,38 & 4,24 \\
\hline \multicolumn{8}{|c|}{ Bulls $(n=24)-$ worsens sons $(n=61)$} \\
\hline 1. $\bar{x} \pm \mathrm{S}_{\overline{\mathrm{x}}}$ & $185,5 \pm 1,1$ & $366,3 \pm 1,7$ & $848 \pm 6$ & $8,1 \pm .1$ & $49,4 \pm .2$ & $38,8 \pm .3$ & $98 \pm .4$ \\
\hline 2. $\mathrm{C}_{\mathrm{V}}$ & 8,78 & 7,14 & 11,25 & 12,74 & 7,38 & 12,48 & 5,5 \\
\hline \multicolumn{8}{|c|}{ HEREFORD BREED } \\
\hline \multicolumn{8}{|c|}{$\begin{array}{c}\text { Hereford breed in general } \\
\text { Bulls }(\mathrm{n}=15) \text { - improvers of sons }(\mathrm{n}=47)\end{array}$} \\
\hline 1. $\bar{x} \pm \mathrm{S}_{\overline{\mathrm{x}}}$ & $220,3 \pm 1,5$ & $439,6 \pm 2,2$ & $1029 \pm 8$ & $6,6 \pm .1$ & $54,3 \pm .2$ & $45,5 \pm .3$ & $102,8 \pm .4$ \\
\hline 2. $\mathrm{C}_{\mathrm{V}}$ & 14,25 & 10,33 & 14,85 & 14,55 & 8,03 & 11,8 & 8,24 \\
\hline \multicolumn{8}{|c|}{ Bulls $(n=5)-$ neutrals by sons $(n=22)$} \\
\hline 1. $x \pm \mathrm{S}_{-}$ & $221 \pm 2,4$ & $432,7 \pm 3,7$ & $1012 \pm 12$ & $6,5 \pm .1$ & $53,3 \pm 4$ & $45 \pm .4$ & $99,9 \pm .7$ \\
\hline 2. $\mathrm{C}_{\mathrm{V}}$ & 13,45 & 10,42 & 14,44 & 15,7 & 8,27 & 11,33 & 8,76 \\
\hline \multicolumn{8}{|c|}{ Bulls $(n=12)-$ worsens sons $(n=41)$} \\
\hline 1. $\bar{x} \pm \mathrm{S}_{\overline{\mathrm{x}}}$ & $221,3 \pm 1,6$ & $423,9 \pm 2,4$ & $953 \pm 8$ & $6,9 \pm .1$ & $52,3 \pm .3$ & $42,6 \pm .4$ & $96,8 \pm .5$ \\
\hline 2. $\mathrm{C}_{\mathrm{V}}$ & 13,98 & 10,67 & 15,3 & 13,8 & 9,61 & 15,65 & 8,87 \\
\hline
\end{tabular}

The interconnection of the signs of evaluating the calves according to their own productivity (Table 2 ) shows that the nature of these correlations is very labile, but it gives a complete picture of the general biological laws, as a single system of all body properties. It should be noted that the live weight of gobies at 8 months of age has the highest correlation with the live weight of 15 months. $(0.502-0.701)$. This indicates that the milk yield of mothers and the live weight of gobies at the age of 15 months are characterized by an average, direct and significant dependence. But with other signs, the milkiness of mother cows (live weight of gobies at 8 months) has a weak connection (-0.276-0.428). A high relationship is found in live weight of gobies at the age of 15 months and average daily growth in the period from 8 to 15 months with all the other signs of evaluating the calves according to their own productivity. An exception to this is the connection with feed costs, which is characterized by negative values $(\mathrm{P}>0.99-0.999)$.

Table 2. The relationship of the signs of the assessment of gobies of Kalmyk and Hereford breeds by their own productivity, grown on farms of various categories

\begin{tabular}{|c|c|c|c|c|c|c|c|c|c|}
\hline \multirow{2}{*}{$\begin{array}{l}\text { Category } \\
\text { of bulls }\end{array}$} & \multirow{2}{*}{ Signs } & \multicolumn{8}{|c|}{ Signs } \\
\hline & & $1 * *$ & $2 * *$ & $3 * *$ & $4 * *$ & $5 * *$ & $6^{* *}$ & $7 * *$ & $8 * *$ \\
\hline \multicolumn{10}{|c|}{ Kalmyk and Hereford breed $*$} \\
\hline \multicolumn{10}{|c|}{ Tribal plants } \\
\hline \multirow{8}{*}{ U1 } & $1 * *$ & - & .142 &.-102 & .165 & .365 & -.143 & .149 & -.007 \\
\hline & $2 * *$ & .722 & - & .244 & -.330 & -.235 & -.284 & .382 & .666 \\
\hline & $3 * *$ & .090 & .748 & - & +.055 & -.448 & -.206 & .548 & .627 \\
\hline & $4 * *$ & -.146 & -.598 & -.730 & - & .192 & .140 & -.653 & -.648 \\
\hline & $5 * *$ & .281 & .383 & .287 & -.196 & - & -.154 & -.015 & -.362 \\
\hline & $6 * *$ & .336 & .762 & .785 & -.661 & .562 & - & -.367 & -.489 \\
\hline & $7 * *$ & .224 & .671 & .779 & -.796 & .572 & .739 & - & .870 \\
\hline & $8 * *$ & .453 & .894 & .863 & -.777 & .573 & .911 & .884 & - \\
\hline $\mathrm{N}$ & 1 & - & .579 & -.018 & .165 & .001 & .251 & .125 & .542 \\
\hline
\end{tabular}




\begin{tabular}{|c|c|c|c|c|c|c|c|c|c|}
\hline & 2 & .553 & - & .513 & .125 & -.125 & .205 & .493 & .850 \\
\hline & 3 & -.063 & .629 & - & .212 & -.166 & .074 & .406 & .581 \\
\hline & 4 & .065 & -.433 & -.700 & - & -.034 & -.071 & -.572 & -.138 \\
\hline & 5 & .279 & .362 & .281 & -.072 & - & .202 & .386 & .163 \\
\hline & 6 & .285 & .705 & .788 & -.690 & .529 & - & .253 & .477 \\
\hline & 7 & .017 & .601 & .769 & -.685 & .586 & .821 & - & .789 \\
\hline & 8 & .252 & .793 & .881 & -.739 & .554 & .942 & .928 & - \\
\hline \multirow{8}{*}{ Ux } & 1 & - & .572 & -.494 & .121 & .102 & .075 & -.175 & -.185 \\
\hline & 2 & .733 & - & .431 & -.167 & .013 & .428 & .369 & .633 \\
\hline & 3 & .113 & .753 & - & -.310 & -.098 & -.372 & .582 & .874 \\
\hline & 4 & .-054 & -.519 & -.706 & - & .259 & .080 & -.325 & -.496 \\
\hline & 5 & .367 & .454 & .326 & -.137 & - & -.190 & -.014 & -.248 \\
\hline & 6 & .400 & .822 & .822 & -.649 & .653 & - & .140 & .518 \\
\hline & 7 & .218 & .677 & .795 & -.692 & .583 & .823 & - & .753 \\
\hline & 8 & .472 & .897 & .865 & -.716 & .621 & .956 & .837 & - \\
\hline \multicolumn{10}{|c|}{ Tribal farms } \\
\hline \multirow{8}{*}{ Ul } & 1 & - & .514 & -.497 &.-699 & .210 & -.005 & -.290 & .116 \\
\hline & 2 & -.009 & - & 460 &.-693 & .537 & .716 & .622 & .862 \\
\hline & 3 & -.254 & .701 & - & .033 & .320 & .748 & .916 & .744 \\
\hline & 4 & .192 & -.550 & -.801 & - & -.408 & -.413 & -.102 & -.514 \\
\hline & 5 & -.363 & .435 & .651 & -.561 & - & .654 & .533 & .726 \\
\hline & 6 & -.142 & .633 & .900 & -.826 & .747 & - & .886 & .955 \\
\hline & 7 & -.179 & .562 & .799 & -.676 & .579 & .690 & - & .884 \\
\hline & 8 & -.283 & .735 & .952 & -.865 & .782 & .935 & .839 & - \\
\hline \multirow{8}{*}{$\mathrm{N}$} & 1 & - & .675 & -.273 & -.753 & .124 & .250 & -.034 & .385 \\
\hline & 2 & .825 & - & .493 & -.811 & .465 & .839 & .672 & .915 \\
\hline & 3 & -.216 & .374 & - & -.179 & .411 & .809 & .904 & .737 \\
\hline & 4 & .582 & .069 & -.835 & - & -.171 & -.874 & -.325 & -.872 \\
\hline & 5 & .272 & .206 & -.090 & .077 & - & .637 & .653 & .662 \\
\hline & 6 & .294 & .659 & .656 & -.424 & .418 & - & .922 & .976 \\
\hline & 7 & .049 & .587 & .933 & -.751 & .157 & .817 & - & .891 \\
\hline & 8 & .117 & .633 & .902 & -.692 & .220 & .887 & .990 & - \\
\hline \multirow{8}{*}{ Ux } & 1 & - & .541 & -.245 & -.465 & .091 & .179 & -.031 & .250 \\
\hline & 2 & .575 & - & .631 & -.694 & .428 & .820 & .753 & .897 \\
\hline & 3 & -.192 & .667 & - & -.375 & .381 & Ю787 & .879 & .805 \\
\hline & 4 & -.091 & -.692 & -.832 & - & -.191 & -.593 & -.461 & -.674 \\
\hline & 5 & .416 & .883 & .692 & -.672 & - & .674 & .681 & .662 \\
\hline & 6 & .206 & .880 & .888 & -.860 & .893 & - & .920 & .968 \\
\hline & 7 & .279 & .704 & .654 & -.728 & .691 & .722 & - & .939 \\
\hline & 8 & .322 & .921 & .849 & -.876 & .912 & .972 & .834 & - \\
\hline \multicolumn{10}{|c|}{ Overall by breed } \\
\hline \multirow{8}{*}{ Ul } & 1 & - & .502 & -.064 & -.203 & .071 & .108 & -.276 & .160 \\
\hline & 2 & .654 & - & .793 & -.433 & .497 & .767 & .395 & .858 \\
\hline & 3 & .016 & .749 & - & -.241 & .519 & .812 & .620 & .867 \\
\hline & 4 & -.056 & -.576 & -.744 & - & -.295 & -.501 & -.298 & -.549 \\
\hline & 5 & .210 & .392 & .335 & -.242 & - & .642 & .489 & .728 \\
\hline & 6 & .255 & .764 & .804 & -.687 & .585 & - & .685 & .947 \\
\hline & 7 & .125 & .649 & .783 & -.693 & .504 & .722 & - & .721 \\
\hline & 8 & .338 & .875 & .884 & -.788 & .596 & .919 & .876 & - \\
\hline
\end{tabular}




\begin{tabular}{|c|c|c|c|c|c|c|c|c|c|}
\hline \multirow{8}{*}{$\mathrm{N}$} & 1 & - & .669 & .217 & .052 & .100 & .221 & -.102 & .357 \\
\hline & 2 & .588 & - & .760 & -.200 & .540 & .721 & .421 & .873 \\
\hline & 3 & -.088 & .600 & - & .040 & .457 & .719 & .676 & .838 \\
\hline & 4 & .120 & -.389 & -.704 & - & -.309 & -.444 & -.255 & -.357 \\
\hline & 5 & .273 & .352 & .255 & -.071 & - & .666 & .402 & .722 \\
\hline & 6 & .283 & .700 & .772 & -.673 & .525 & - & .731 & .936 \\
\hline & 7 & .019 & .597 & .873 & -.687 & .558 & .818 & - & .738 \\
\hline & 8 & .241 & .782 & .876 & -.731 & .541 & .939 & .932 & - \\
\hline \multirow{8}{*}{ Ux } & 1 & - & .701 & .198 & -.157 & .076 & .282 & .040 & .428 \\
\hline & 2 & .722 & - & .773 & -.399 & .401 & .764 & .505 & .887 \\
\hline & 3 & .086 & .742 & - & -.195 & .368 & .754 & .646 & .820 \\
\hline & 4 & -.059 & -.526 & -.723 & - & -.253 & -.531 & -.353 & -.535 \\
\hline & 5 & .377 & .516 & .398 & -.243 & - & .607 & .475 & .625 \\
\hline & 6 & .378 & .826 & .837 & -.687 & .702 & - & .794 & .951 \\
\hline & 7 & .236 & .672 & .770 & -.696 & .603 & .798 & - & .786 \\
\hline & 8 & .459 & .894 & .863 & -.739 & .682 & .959 & .884 & - \\
\hline
\end{tabular}

Note: Ul - improver, $\mathrm{N}$ - neutral, $\mathrm{Ux}$ - deteriorator

* - correlation coefficients located on the diagonal above are determined by the Kalmyk, and below - Hereford rocks

** 1 - live weight in 8;2 - at the age of 15 months; 3 - average daily increase in the period from 8 to 15 months; 4 - feed costs per $1 \mathrm{~kg}$ of growth; 5 - intravital assessment of meat qualities; 6 - general mark; 7 - a comprehensive assessment index for the growing period from 8 to 15 months of age; 8 is a new factor.

The data presented show that by selecting calves by live weight or by growth intensity, we can expect that the cost of feed for growth will decrease, in meat form it will improve.

Allocated by us on the basis of factor analysis, a new factor that summarizes all the signs of evaluating the calves by their own productivity has the property of the greatest conjugation with all the signs. Moreover, the higher correlation of the new factor with the signs of assessment, as well as with the complex index, as well as with the general assessment, allows it to be used as a selection trait (Table 3).

Table 3. The relationship of the new factor with the signs of the assessment of the bulls on their own productivity

\begin{tabular}{|c|c|c|c|c|c|c|c|}
\hline \multirow{2}{*}{$\begin{array}{c}\text { Category } \\
\text { of bulls }\end{array}$} & \multicolumn{2}{|c|}{$\begin{array}{l}\text { Live weight, } \\
\text { month }\end{array}$} & \multirow{2}{*}{$\begin{array}{c}\text { Average } \\
\text { daily } \\
\text { increase } \\
\text { from } 8 \text { to } \\
15 \\
\text { months }\end{array}$} & \multirow{2}{*}{$\begin{array}{c}\text { Feed } \\
\text { costs per } \\
1 \mathrm{~kg} \text { of } \\
\text { growth }\end{array}$} & \multirow{2}{*}{$\begin{array}{c}\text { Evaluation } \\
\text { of meat } \\
\text { forms }\end{array}$} & \multirow{2}{*}{$\begin{array}{c}\text { Overall } \\
\text { point } \\
\text { grade }\end{array}$} & \multirow{2}{*}{$\begin{array}{l}\text { Integrated } \\
\text { Index }\end{array}$} \\
\hline & 8 & 15 & & & & & \\
\hline \multicolumn{8}{|c|}{ KALMYK BREED } \\
\hline U1 & 0,160 & 0,858 & 0,867 & $-0,549$ & 0,728 & 0,947 & 0,721 \\
\hline $\mathrm{N}$ & 0,357 & 0,873 & 0,838 & $-0,357$ & 0,722 & 0,936 & 0,738 \\
\hline Ux & 0,428 & 0,887 & 0,820 & $-0,535$ & 0,625 & 0,951 & 0,786 \\
\hline \multicolumn{8}{|c|}{ HEREFORD BREED } \\
\hline Ul & 0,388 & 0,875 & 0,884 & $-0,788$ & 0,596 & 0,919 & 0,876 \\
\hline $\mathrm{N}$ & 0,241 & 0,782 & 0,876 & $-0,731$ & 0,541 & 0,939 & 0,932 \\
\hline Ux & 0,459 & 0,894 & 0,863 & $-0,739$ & 0,682 & 0,959 & 0,884 \\
\hline
\end{tabular}


Of great importance in the study of correlation relationships is the method of regression analysis, which with a certain degree of probability allows us to predict the value of one attribute by the value of another. We have compiled breeding indices based on regression equations to determine the value of a new factor in the breeding value of bulls at 8,15 months of age in terms of live weight and average daily growth from 8 to 15 months (tab. 4 , $5)$.

Table 4. Breeding indices for determining the breeding value of calves by live weight at 8,15 months of age and average daily gain for a period from 8 to 15 months different breeds grown on farms of various categories

\begin{tabular}{|c|c|c|c|}
\hline \multirow{2}{*}{$\begin{array}{l}\text { Fathers } \\
\text { Category }\end{array}$} & \multicolumn{2}{|c|}{ Live weight, $\mathrm{kg}$ in age } & \multirow{2}{*}{$\begin{array}{l}\text { The average daily } \\
\text { increase in the period } \\
\text { from } 8 \text { to } 15 \text { months, } g \\
\left(\mathrm{X}_{3}\right)\end{array}$} \\
\hline & 8 month $\left(X_{1}\right)$ & 15 months $\left(\mathrm{X}_{2}\right)$ & \\
\hline \multicolumn{4}{|c|}{ KALMYK BREED. Tribal plants } \\
\hline $\mathrm{Ul}$ & $-0,001 x_{1}+.25$ & $0,056 x_{2}-19,66$ & $0,022 x_{3}-17,41$ \\
\hline $\mathrm{N}$ & $0,061 \mathrm{x}_{1}-10,85$ & $0,117 x_{2}-40,19$ & $0,028 x_{3}-21,86$ \\
\hline Ux & $-0,029 x_{1}+5,28$ & $0,105 x_{2}-35,84$ & $0,032 x_{3}-24,96$ \\
\hline \multicolumn{4}{|c|}{ Tribal farms } \\
\hline Ul & $0,007 \mathrm{x}_{1}-1,37$ & $0,051 x_{2}-19,38$ & $0,010 x_{3}-8,59$ \\
\hline $\mathrm{N}$ & $-0,022 x_{1}-4,19$ & $0,049 x_{2}-18,25$ & $0,010 \mathrm{x}_{3}-8,33$ \\
\hline Ux & $-0,020 \mathrm{x}_{1}-3,73$ & $0,054 x_{2}-19,65$ & $0,011 \mathrm{x}_{3}-9,54$ \\
\hline \multicolumn{4}{|c|}{ Tribal farms } \\
\hline $\mathrm{Ul}$ & $0,005 \mathrm{x}_{1}-.878$ & $0,026 x_{2}-11,47$ & $0,009 x_{3}-8,80$ \\
\hline $\mathrm{N}$ & $-0,021 x_{1}-3,80$ & $0,032 \mathrm{x}_{2}-12,05$ & $0,008 x_{3}-7,04$ \\
\hline Ux & $-0,024 \mathrm{x}_{1}-4,46$ & $0,028 \mathrm{x}_{2}-10,51$ & $0,007 \mathrm{x}_{3}-6,05$ \\
\hline \multicolumn{4}{|c|}{ By breed } \\
\hline Ul & $0,009 \mathrm{x}_{1}-1,76$ & $0,032 x_{2}-12,44$ & $0,008 x_{3}-7,40$ \\
\hline $\mathrm{N}$ & $-0,018 \mathrm{x}_{1}-3,31$ & $0,033 x_{2}-12,17$ & $0,008 x_{3}-7,50$ \\
\hline $\mathrm{Ux}$ & $-0,026 \mathrm{x}_{1}-4,81$ & $0,094 x_{2}-12,42$ & $0,009 \mathrm{x}_{3}-7,29$ \\
\hline \multicolumn{4}{|c|}{ HEREFORD BREED. Tribal plants } \\
\hline Ul & $0,015 x_{1}-3,42$ & $0,020 x_{2}-8,73$ & $0,006 \mathrm{x}_{3}-6,01$ \\
\hline $\mathrm{N}$ & $0,009 \mathrm{x}_{1}-2,04$ & $0,018 x_{2}-7,69$ & $0,006 \mathrm{x}_{3}-6,09$ \\
\hline Ux & $-0,016 x_{1}-3,68$ & $0,020 \mathrm{x}_{2}-8,65$ & $0,006 \mathrm{x}_{3}-5,84$ \\
\hline \multicolumn{4}{|c|}{ Tribal farms } \\
\hline Ul & $-0,008 x_{1}+1,46$ & $0,018 x_{2}-7,84$ & $0,006 \mathrm{x}_{3}-5,56$ \\
\hline $\mathrm{N}$ & $0,002 \mathrm{x}_{1}-.55$ & $0,012 x_{2}-5,36$ & $0,006 x_{3}-6,19$ \\
\hline $\mathrm{Ux}$ & $0,009 \mathrm{x}_{1}-1,85$ & $0,023 \mathrm{x}_{2}-9,12$ & $0,005 x_{3}-4,92$ \\
\hline \multicolumn{4}{|c|}{ By breed } \\
\hline Ul & $0,011 \mathrm{x}_{1}-2,37$ & $0,019 \mathrm{x}_{2}-8,44$ & $0,006 x_{3}-5,93$ \\
\hline $\mathrm{N}$ & $0,008 \mathrm{x}_{1}-1,79$ & $0,017 x_{2}-7,48$ & $0,006 x_{3}-6,04$ \\
\hline $\mathrm{Ux}$ & $0,015 x_{1}-3,27$ & $0,020 \mathrm{x}_{2}-8,34$ & $0,006 \mathrm{x}_{3}-5,62$ \\
\hline
\end{tabular}

Table 5. Breeding indices for determining the breeding value of gobies of different breeds raised in farms of various categories

\begin{tabular}{|c|c|c|c|}
\hline $\begin{array}{c}\text { Fathers } \\
\text { Category }\end{array}$ & $\begin{array}{c}\text { Live weight }(\mathrm{kg}) \\
\text { at } 8\left(\mathrm{x}_{1}\right) \text { and } 15 \\
\left(\mathrm{x}_{2}\right) \text { months of age }\end{array}$ & $\begin{array}{c}\text { Live weight }(\mathrm{kg}) \text { at } 15 \text { and } \\
\text { the average daily gain }(\mathrm{g}) \\
\text { in the period of } 8-15 \\
\text { months }\left(\mathrm{x}_{3}\right)\end{array}$ & $\begin{array}{c}\text { Live weight at } 8,15 \\
\text { months of age and average } \\
\text { daily gain in the period of } \\
8-15 \text { months }\end{array}$ \\
\hline \multicolumn{3}{|c|}{ KALMYK BREED. Tribal plants } \\
\hline
\end{tabular}




\begin{tabular}{|c|c|c|c|}
\hline Ul & $\begin{array}{c}-0,021 \mathrm{x}_{1}+.058 \mathrm{x}_{2} \\
-16,26\end{array}$ & $0,046 x_{2}+.017 x_{3}-29,82$ & $\begin{array}{c}-0,007 \mathrm{x}_{1}+0,047 \mathrm{x}_{2}+ \\
0,017 \mathrm{x}_{3}-28,55\end{array}$ \\
\hline $\mathrm{N}$ & $\begin{array}{c}0,009 \mathrm{x}_{1}+.111 \mathrm{x}_{2} \\
-39,63\end{array}$ & $0,103 x_{2}+.009 x_{3}-42,82$ & $\begin{array}{c}0,024 \mathrm{x}_{1}+0,080 \mathrm{x}_{2}+ \\
0,014 \mathrm{x}_{3}-42,14\end{array}$ \\
\hline Ux & $\begin{array}{c}-0,129 \mathrm{x}_{1}+.018 \mathrm{x}_{2} \\
-38,95\end{array}$ & $0,052 x_{2}+.027 x_{3}-38,94$ & $\begin{array}{c}-2,417 \mathrm{x}_{1}+2,468 \mathrm{x}_{2}- \\
0,480 \mathrm{x}_{3}-39,47\end{array}$ \\
\hline \multicolumn{4}{|c|}{ Tribal farms } \\
\hline U1 & $\begin{array}{c}-0,028 \mathrm{x}_{1}+.065 \mathrm{x}_{2} \\
-19,27 \\
\end{array}$ & $0,039 x_{2}+.006 x_{3}-19,92$ & $\begin{array}{c}-0,005 \mathrm{x}_{1}+0,044 \mathrm{x}_{2}+ \\
0,005 \mathrm{x}_{3}-19,80 \\
\end{array}$ \\
\hline $\mathrm{N}$ & $\begin{array}{c}-0,025 \mathrm{x}_{1}+.065 \mathrm{x}_{2} \\
-19,36 \\
\end{array}$ & $0,039 x_{2}+.005 x_{3}-18,80$ & $\begin{array}{c}-0,003 \mathrm{x}_{1}+0,042 \mathrm{x}_{2}+ \\
0,004 \mathrm{x}_{3}-18,88 \\
\end{array}$ \\
\hline $\mathrm{Ux}$ & $\begin{array}{c}-0,027 \mathrm{x}_{1}+.065 \mathrm{x}_{2} \\
-18,60\end{array}$ & $0,039 x_{2}+.006 x_{3}-18,87$ & $\begin{array}{c}-0,001 \mathrm{x}_{1}+0,040 \mathrm{x}_{2}+ \\
0,005 \mathrm{x}_{3}-18,85\end{array}$ \\
\hline \multicolumn{4}{|c|}{ Tribal farms } \\
\hline Ul & $\begin{array}{c}-0,038 \mathrm{x}_{1}+.047 \mathrm{x}_{2} \\
-11,89 \\
\end{array}$ & $0,002 x_{2}+.008 x_{3}-9,14$ & $\begin{array}{c}-0,031 \mathrm{x}_{1}+0,038 \mathrm{x}_{2}+ \\
0,002 \mathrm{x}_{3}-11,29 \\
\end{array}$ \\
\hline $\mathrm{N}$ & $\begin{array}{c}-0,017 \mathrm{x}_{1}+.042 \mathrm{x}_{2} \\
-13,03\end{array}$ & $0,016 x_{2}+.005 x_{3}-10,27$ & $\begin{array}{c}-0,003 \mathrm{x}_{1}+0,019 \mathrm{x}_{2}+ \\
0,004 \mathrm{x}_{3}-10,63\end{array}$ \\
\hline $\mathrm{Ux}$ & $\begin{array}{c}-0,021 \mathrm{x}_{1}+.039 \mathrm{x}_{2} \\
-10,68 \\
\end{array}$ & $0,019 x_{2}+.003 x_{3}-9,63$ & $\begin{array}{c}-0,023 \mathrm{x}_{1}+0,045 \mathrm{x}_{2}- \\
0,001 \mathrm{x}_{3}-10,88 \\
\end{array}$ \\
\hline \multicolumn{4}{|c|}{ By breed } \\
\hline $\mathrm{Ul}$ & $\begin{array}{c}-0,021 \mathrm{x}_{1}+.039 \mathrm{x}_{2} \\
-11,10 \\
\end{array}$ & $0,017 \mathrm{x}_{2}+.005 \mathrm{x}_{3}-10,95$ & $\begin{array}{c}-0,013 \mathrm{x}_{1}+0,030 \mathrm{x}_{2}+ \\
0,002 \mathrm{x}_{3}-10,95 \\
\end{array}$ \\
\hline $\mathrm{N}$ & $\begin{array}{c}-0,021 \mathrm{x}_{1}+.043 \mathrm{x}_{2} \\
-12,21\end{array}$ & $0,021 x_{2}+.004 x_{3}-11,50$ & $\begin{array}{c}-0,015 \mathrm{x}_{1}+0,035 \mathrm{x}_{2}+ \\
0,002 \mathrm{x}_{3}-11,86\end{array}$ \\
\hline Ux & $\begin{array}{c}-0,024 \mathrm{x}_{1}+.044 \mathrm{x}_{2} \\
-11,83\end{array}$ & $0,024 x_{2}+.004 x_{3}-11,78$ & $\begin{array}{c}-0,023 \mathrm{x}_{1}+0,043 \mathrm{x}_{2}+ \\
0,001 \mathrm{x}_{3}-11,81 \\
\end{array}$ \\
\hline \multicolumn{4}{|c|}{ HEREFORD BREED. Tribal plants } \\
\hline Ul & $\begin{array}{c}-0,014 \mathrm{x}_{1}+.026 \mathrm{x}_{2} \\
-8,54 \\
\end{array}$ & $0,012 x_{2}+.003 x_{3}-8,58$ & $\begin{array}{c}0,012 \mathrm{x}_{1}+0,001 \mathrm{x}_{2}+ \\
0,006 \mathrm{x}_{3}-8,63 \\
\end{array}$ \\
\hline $\mathrm{N}$ & $\begin{array}{c}-0,010 \mathrm{x}_{1}+.021 \mathrm{x}_{2} \\
-6,96 \\
\end{array}$ & $0,009 x_{2}+.004 x_{3}-8,21$ & $\begin{array}{c}0,007 \mathrm{x}_{1}+0,005 \mathrm{x}_{2}+ \\
0,005 \mathrm{x}_{3}-8,79 \\
\end{array}$ \\
\hline $\mathrm{Ux}$ & $\begin{array}{c}-0,014 \mathrm{x}_{1}+.027 \mathrm{x}_{2} \\
-8,35\end{array}$ & $0,013 x_{2}+.003 x_{3}-8,43$ & $\begin{array}{c}0,011 \mathrm{x}_{1}+0,002 \mathrm{x}_{2}+ \\
0,005 \mathrm{x}_{3}-8,50\end{array}$ \\
\hline \multicolumn{4}{|c|}{ Tribal farms } \\
\hline Ul & $\begin{array}{c}-0,015 \mathrm{x}_{1}+.021 \mathrm{x}_{2} \\
-5,93 \\
\end{array}$ & $-0,001 x_{2}+.005 x_{3}-5,53$ & $\begin{array}{c}0,004 \mathrm{x}_{1}-0,004 \mathrm{x}_{2}+ \\
0,006 \mathrm{x}_{3}-5,62\end{array}$ \\
\hline $\mathrm{N}$ & $\begin{array}{c}-0,026 \mathrm{x}_{1}+.032 \mathrm{x}_{2} \\
-8,23 \\
\end{array}$ & $0,007 x_{2}+.005 x_{3}-8,22$ & $\begin{array}{c}-1,186 \mathrm{x}_{1}+1,192 \mathrm{x}_{2}+ \\
.244 \mathrm{x}_{3}-8,29 \\
\end{array}$ \\
\hline $\mathrm{Ux}$ & $\begin{array}{c}-0,009 \mathrm{x}_{1}+.027 \mathrm{x}_{2} \\
-9,10\end{array}$ & $0,016 x_{2}+.003 x_{3}-8,78$ & $\begin{array}{c}0,014 \mathrm{x}_{1}+0,001 \mathrm{x}_{2}+ \\
0,006 \mathrm{x}_{3}-8,41\end{array}$ \\
\hline \multicolumn{4}{|c|}{ By breed } \\
\hline $\mathrm{Ul}$ & $\begin{array}{c}-0,013 \mathrm{x}_{1}+.025 \mathrm{x}_{2} \\
-8,16\end{array}$ & $0,011 x_{2}+.003 x_{3}-8,17$ & $\begin{array}{c}0,007 \mathrm{x}_{1}+0,004 \mathrm{x}_{2}+ \\
0,005 \mathrm{x}_{3}-8,20\end{array}$ \\
\hline $\mathrm{N}$ & $\begin{array}{c}-0,011 \mathrm{x}_{1}+.022 \mathrm{x}_{2} \\
-6,88 \\
\end{array}$ & $0,009 x_{2}+.004 x_{3}-8,21$ & $\begin{array}{c}0,006 \mathrm{x}_{1}+0,005 \mathrm{x}_{2}+ \\
0,005 \mathrm{x}_{3}-8,67 \\
\end{array}$ \\
\hline Ux & $\begin{array}{c}-0,013 \mathrm{x}_{1}+.028 \mathrm{x}_{2} \\
-8,19\end{array}$ & $0,012 x_{2}+.003 x_{3}-8,16$ & $\begin{array}{c}0,013 \mathrm{x}_{1}+0,001 \mathrm{x}_{2}+ \\
0,006 \mathrm{x}_{3}-8,15\end{array}$ \\
\hline
\end{tabular}


Substituting their values in the selection indices, taking into account which father this goby comes from (improver, neutral, deteriorator), we obtain a new factor. If they are positive, then the goby is evaluated according to its own productivity, Ul, if it is negative, Ux.

\section{Conclusion}

The proposed breeding indices on one basis are quite suitable for the preliminary selection of calves in commodity farms or in pedigree breeding for beef cattle. In order to get a final assessment, we compiled new breeding indices, including two (live weight at 8 and 15 months., Live weight at 15 months. And average daily growth in the period from 8 to 15 months.), As well as three characteristics (live weight in 8, 15 months and average daily gain). These signs are objective, easy to account. According to the estimates obtained for individual bull-sons, it is possible by summing up the values of new factors to identify the bulls' assessment of the quality of the offspring. Analysis of the assessment materials is characterized by high reliability $(\mathrm{P}>0.999)$.

Thus, using factor analysis and the multiple linear regression method, the breeder's subjectivity, difficulties in accounting for feed intake, etc. are completely eliminated. The whole work is simplified. The assessment factors obtained by two or three characteristics most fully characterize the breeding value of bulls and bulls, compared to assessment on one basis, and they should probably be used in those farms where in-depth pedigree work (breeding plants) is conducted. Such breeding indices can be developed by assessing the heifers' own productivity and, on this basis, select producers who give high-quality sons and daughters. In this case, the selection will be based on theoretical, sound assumptions, and not on the intuition of breeders, which allows you to turn selection into a technological process with its strict regulation. The selection process will become collective creativity. Technologicalization of it will allow equipping specialists with a reliable means of increasing the productive and fiery qualities of beef cattle. Breeding will turn from art into a breeder's tool.

\section{References}

1. A. Kruglyak, T. Kruglyak, Actual problems of the intensive development of animal breeding, 22-1, 107 (2019)

2. A. Zelenkov, P. Zelenkov, Dairy and meat cattle breeding, 1, 21 (2014)

3. K. Dzhulamanov, N. Gerasimov, Herald of beef cattle breeding, 1, 63, 25 (2010)

4. P. Zelenkov, A. Zelenkov, G. Zelenkova, Veterinarnaya patologiya, 3-4(49-50), 15 (2014)

5. A. Zelenkov, P. Zelenkov, G. Zelenkova, Vestnik of Don state agrarian university, 4-1 (14), 35 (2014)

6. A. Zelenkov, G. Zelenkova, A. Ermakov, A. Pakhomov, In the collection: State and prospects for the development of the agricultural complex. collection of scientific papers of the XII International Scientific and Practical Conference in the framework of the XXII Agro-Industrial Forum of the South of Russia and the Interagromash exhibition (Rostov-on-Don), 856 (2019)

7. M. Karanin, P. Zelenkov, A. Zelenkov, In the collection: Innovative technologies in animal husbandry. materials of the Interuniversity student scientific-practical conference, 192 ( 2015) 
8. P. Zelenkov, A. Zelenkov, In the collection: Selection of farm animals and livestock production technology. Materials of the international scientific-practical conference, 26 (2016)

9. V. Chernomyrdin, F. Kayumov, L. Mayevskaya, Herald of beef cattle breeding, 1, 63, 44 (2010)

10. P. Zelenkov, A. Zelenkov, Collection of scientific papers of the Stavropol Research Institute of Animal Husbandry and Feed Production (Stavropol), 377 (2013)

11. S. Makayev, M. Abbasov, Herald of beef cattle breeding, 3, 64, 34 (2011)

12. V. Chernomyrdin, F. Kayumov, L. Mayevskaya, Herald of beef cattle breeding, 1(75), 21 (2012) 